\title{
Semantic Interpretation of Prepositions for NLP Applications
}

\author{
Sven Hartrumpf Hermann Helbig Rainer Osswald \\ Intelligent Information and Communication Systems (IICS) \\ University of Hagen (FernUniversität in Hagen) \\ 58084 Hagen, Germany \\ Firstname.Lastname@fernuni-hagen.de
}

\begin{abstract}
The proper interpretation of prepositions is an important issue for automatic natural language understanding. We present an approach towards PP interpretation as part of a natural language understanding system which has been successfully employed in various NLP tasks for information retrieval and question answering. Our approach is based on the so-called MultiNet paradigm, a knowledge representation formalism especially designed for the representation of natural language semantics. The paper describes how the information about the semantic interpretation of PPs is represented in the lexicon and in PP interpretation rules and how this information is used during semantic analysis. Moreover, we report on experiments that evaluate the impact of using this information about PP interpretation on the CLEF question answering task.
\end{abstract}

\section{Introduction}

Advanced NLP applications such as question answering require deep semantic interpretation. In this context, prepositions play an important role since they encode relational information. The proper semantic analysis of prepositional phrases is faced with various problems: (1) There is the well-known problem of attachment ambiguities. (2) Prepositions are highly polysemous, i.e. their interpretation is typically context dependent. (3) Prepositions often occur in collocations, where their interpretation is irregular.

Although a large amount of work in the NLP community has focused on resolving attachment ambiguities, there are only first steps towards a systematic description of preposition semantics which has sufficient coverage for NLP appli- cations (Litkowski and Hargraves, 2005; SaintDizier, 2005). The automatic interpretation of prepositions in English has been tackled, for example, by Litkowski (2002), who presents handcrafted disambiguation rules, and O'Hara and Wiebe (2003), who propose a statistical approach based on collocations. However, in order to be applicable for semantic inference, the representation of preposition semantics should ideally be integrated within a full-fledged knowledge representation formalism.

In spite of the broad linguistic investigations on preposition semantics, ${ }^{1}$ the corresponding results have seldom found their way into real NLP applications. Information retrieval systems, on the other hand, which claim to use NLP techniques often do not cope with the semantic content of prepositions at all (even if they bear the term semantic in their title, as with Latent Semantic Analysis (Letsche and Berry, 1997)). In many cases prepositions are even dropped as stop words in such systems. If one really wants to syntactico-semantically analyze texts and derive formal semantic representations, the interpretation of prepositions and especially the disambiguation of their different readings is a central problem in Indoeuropean languages like English, French, Russian, and German.

In this paper we describe the semantic treatment of this problem for German, using the knowledge representation formalism of Multilayered Extended Semantic Networks (MultiNet) (Helbig, 2006). The advantage of this approach is its applicability to different languages and different processes of automatic natural language understanding. Since MultiNet complies with the criteria of universality, homogeneity, and interoperability (Helbig, 2006, Chapter 1), it can be used to formalize the semantics of lexemes (Hartrumpf et al.,

\footnotetext{
${ }^{1}$ See also the overview given in (Zelinski-Wibbelt, 1993).
} 
Table 1: Classes of PP interpretation

\begin{tabular}{|c|c|c|}
\hline & complement & adjunct \\
\hline regular & $\begin{array}{l}\text { She lives in } \\
\text { Berlin. }\end{array}$ & $\begin{array}{l}\text { They met in Au- } \\
\text { gust. }\end{array}$ \\
\hline irregula & $\begin{array}{l}\text { He believes in } \\
\text { destiny. }\end{array}$ & $\begin{array}{l}\text { He was killed on } \\
\text { the run. }\end{array}$ \\
\hline
\end{tabular}

2003) as well as that of sentences and texts (see (Leveling and Hartrumpf, 2005), where MultiNet has been employed for semantic annotation of large corpora). It can also be used as a semantic interlingua throughout all NLP modules and all applications of an NLP system (Leveling, 2005). Typical applications that can profit from a precise PP interpretation component are question answering (QA) systems and natural language interfaces.

\section{General Classes of PP Interpretation}

The semantic interpretation of prepositions in NLP has to deal with the following two orthogonal phenomena: regular (or compositional or productive) vs. irregular (or non-compositional or collocative) uses of prepositions and uses in complements vs. uses in adjuncts (putting aside the wellknown issue of borderline cases). ${ }^{2}$ There are thus the four cases indicated in Table 1. In German, PPs can occur also as invariant syntagmas in light verb constructions ('Funktionsverbgefüge') such as in Beschlag nehmen ('to occupy'), to which the complement-adjunct distinction does not apply. In the following, we keep aside the interpretation of prepositions in fixed phrases, which is the case for the light verb constructions just mentioned and also for the irregular adjunct interpretation shown at the lower right of Table 1. This leaves us with three types of PP interpretation: (regular) adjunct interpretation, regular complement interpretation, and irregular complement interpretation.

The standard examples for regular adjunct interpretation are local (or directional) and temporal PPs, which can be attached to verbs, nouns, and adjectives:

\footnotetext{
${ }^{2}$ See also (Rauh, 1993).
}
a. das in Europa beliebte Spiel the in Europe popular game 'the game that is popular in Europe'
b. das im Winter dunkle Haus the in winter dark house 'the house that is dark in winter'

Notice that German, unlike English, allows adjectives with PP adjuncts (or complements) as attributes of nouns, witness (3).

It is characteristic of regular adjunct interpretation that the preposition has a meaning of its own and expresses some sort of relationship. Besides local and temporal specifications, there are of course many other relationships expressed by regular PP adjuncts such as instrumental (4-a), comitative (4-b), and part-whole (4-c) interpretations. $^{3}$
a. John cleaned the floor with his shirt.
b. Mary visited London with her sister.
c. a building with large windows

The examples in (4) furthermore illustrate the well known fact that prepositions are highly polysemous in general. Within our approach described in Sect. 4, prepositions currently have up to sixteen readings. ${ }^{4}$

We speak of a regular complement interpretation if the PP is subcategorized and its interpretation is identical to the (correct) interpretation of the PP when analyzed as an adjunct. These adjunct interpretations of PPs are defined by the set of PP rules, which are explained in Sect. 4.2. Examples of regular complements are wohnen in/auf/... ('to live in/on/...'), schicken nach/in/... ('to send to/into/...'), mitkommen mit ('to come along with'), and Einstieg in ('getting in'). Here, the choice of the preposition in the PP complement of the lexeme is determined by the semantic characterization of the complement.

In the case of irregular complement interpretation, in contrast, the selection of the preposition is an idiosyncratic property of the subcategorizing lexical entry. The preposition alone can be viewed as semantically empty; only the combination of

\footnotetext{
${ }^{3}$ In our approach, 91 different relations occur, often in combinations; see Sect. 4.2.

${ }^{4}$ The average number of readings is 2.44 , which is slightly higher than the polysemy degree $2.27(=847 / 373)$ reported by Litkowski and Hargraves (2005) for English. But our lexicon currently contains only few phrasal (or complex) prepositions like in Anbetracht ('in view of'), which often have only one reading.
} 
the lexeme and the preposition bears semantics. Examples for verbs, adjectives, and nouns of this sort are glauben an ('to belief in'), sich verlassen auf ('to depend on'), gut in ('good at'), and Wut auf ('anger at').

It should be noted, however, that there is a whole spectrum of subregular phenomena within what we called "irregular" complement semantics. ${ }^{5}$ Consider, for instance, the verbs ernennen $z u$, bestimmen zu ('appoint', 'designate'), küren $z u$ ('elect'), and weihen zu ('ordain'). Even if the preposition $z u$ ('to') could be said to semantically express some sort of abstract goal in these cases, an adequate interpretation rule associated with that preposition would have to make reference to a rather restricted semantic class of verbs. We regard it therefore as a matter of lexical semantic organization to capture such subregularities within the interpretation of prepositional complements by means of appropriate semantic verb templates in the lexicon; see (Osswald et al., 2006) for details. In the context of the present paper, an interpretation of a prepositional complement is called irregular if the interpretation is not covered by one of our PP interpretation rules.

Table 2 show the frequency of adjunct and complement interpretations in different corpora. The numbers in the token rows are derived from automatic corpus parses (see below), so there is some noise to be expected, but the trends should be valid.

\section{The Semantic Formalism MultiNet}

MultiNet is one of the few knowledge representation paradigms which have also been used as a semantic interlingua in real-life NLP applications (Leveling and Helbig, 2002). The MultiNet formalism represents meanings of natural language expressions by means of (partial) semantic networks. A semantic network consists of nodes representing concepts and edges representing relations between concepts. Every node is additionally labeled by a sort arising from an ontologically or epistemically motivated classification of concepts (see Appendix, Table 4). Apart from that, every node is embedded in a system of layer attributes and their values expressing the extension type, facticity, genericity, referential determination, quantification, and others. The rela-

\footnotetext{
${ }^{5}$ See also the discussion in (Baldwin, 2005) on prepositional verbs in English.
}

tions connecting the concepts in a semantic network have to be taken from a predefined set of expressional means, which are systematically described and formally characterized (Helbig, 2006). A strongly abbreviated description of all MultiNet relations used in this paper can be found in Table 5 of the Appendix.

For the semantic characterization of the selectional restrictions (i.e. valencies) of lexemes, an additional set of 16 binary semantic features (such as animate, human, artificial, movable, and institution) is provided, which can be combined with the above-mentioned sorts to yield a rich repertoire of semantical characterizations for the description of the slots and fillers corresponding to the valencies. These expressional means have been used in the computational lexicon HaGenLex (see Sect. 4.1).

\section{Resources for PP Interpretation}

Three sources of preposition information are available to the syntactico-semantic parser used in our NLP applications: ${ }^{6}$ subcategorization information in the lexicon, context dependent PP interpretation rules, and an annotated PP corpus.

\subsection{Selection of PPs in the Lexicon}

Our parser makes use of the computational lexicon HaGenLex (Hagen German Lexicon, see (Hartrumpf et al., 2003)), which is a general domain lexicon for German with about 25,000 entries (including 136 prepositions). Each entry contains detailed morpho-syntactic and semantic information. In particular, the lexicon provides valency frames for nouns, verbs, and adjectives (in the lexical feature SELECT). This includes complements that are syntactically realized by a PP. Each complement is characterized by one or more syntactic specifications and its semantic contribution to the head word. This contribution can be a MultiNet relation (case role) or a more complex MultiNet expression directly or indirectly connecting the representation of the complement and of the head, which typically involves other complements.

In order to capture semantic constraints on possible adjuncts, the set of semantic relations compatible with a given lexeme is specified in the lexicon (under the lexical feature COMPAT-R). This information is inherited from the semantic class

\footnotetext{
${ }^{6}$ See (Hartrumpf, 2003, Chap. 3) for a description of the parser.
} 
Table 2: Class frequencies in corpora.

\begin{tabular}{lrrr}
\hline corpus & adjunct & complement & $\begin{array}{l}\text { regular ones among com- } \\
\text { plements (verbs only) }\end{array}$ \\
\hline German QA @ CLEF documents & $80.6 \%$ & $19.4 \%$ & $17.5 \%$ \\
German Wikipedia (March 2005) & $80.1 \%$ & $19.9 \%$ & - \\
\hline
\end{tabular}

of the lexeme so that the set of all possible adjunct readings for a PP (see next section) can be filtered. Lexical entries exemplifying both aspects are listed in Fig. 1.

\subsection{Interpretation Rules for Prepositions}

The second knowledge source for PP interpretation are symbolic $P P$ interpretation rules developed for adjunct interpretations. The premise of such a rule encodes under which semantic and syntactic constraints a specific preposition interpretation is possible; the conclusion specifies a semantic network representing the PP semantics. Two simplified interpretation rules are shown in Fig. 2; the effect of the second rule is depicted in Fig. 3. The rules are licensing possible interpretations; if several rule premises can be unified with a given pair of a preposition's complement and a candidate mother, the PP disambiguation module retreats to a statistical back-off model to resolve this ambiguity. Currently, we have 332 rules.

The interpretation rules can be viewed as a declarative part of the corresponding preposition entry in the lexicon. For maintenance reasons, the rules are stored and manipulated separately. They are linked to the lexicon by lexeme IDs.

The rules show that PP semantics involves many areas of semantics. For example, MultiNet defines around 150 relations and 91 of them are used in the conclusion of PP rules. The 10 most frequent ones are: LOC, VAL, TEMP, ATTR, ELMT, ATTCH, DIRCL, INSTR, SUBM, ORIGM (see Table 5). As exemplified by the second rule of Fig. 2, the semantic network specified in the conclusion of a rule often consists of more than one network edge; on average, an interpretation has 1.69 edges.

\subsection{Annotated PP Corpus}

A third source of preposition information is an annotated PP corpus and statistics derived from it. The occurrences of six frequent prepositions in 840 PPs have been manually annotated with the correct PP attachment and most likely PP interpre- "prahlen.1.1" [ \% 'boast'

verb

syn v-control subjeq

semsel [

$\mathrm{v}$-nonment-action

select $<$

[ agt-select sel semsel sem entity human + ]

[ mcont-select

oblig -

sel syn (mit-dat-pp-syn damit-dass-syn damit-zu-inf-syn)] $\rangle$

compat-r \{dur fin strt\}

example <"(Der Mann) (prahlt) (mit seinen Erfolgen)." $\rangle]]$

\% 'The man boasts his successes.'

"Gegenstück.1.1" [ \% 'counterpart'

n-neut

semsel [

count-n

sem [ entity prot-theor-concept

net /(attch x1 c) (equ c x0)/]

select $<$

[ nselect

sel syn zu-dat-pp-syn] $\rangle$

example $\langle$ "Die Mutter ist das Gegenstück zur Schraube." $\rangle]]$

$\%$ 'The nut is the counterpart to the screw.'

"unabhängig.1.1" [ \% 'independent'

a-nq

semsel [

sem net /(sspe $\mathrm{n} 1 \mathrm{x} 1)(\operatorname{scar} \mathrm{n} 1 \mathrm{x} 0)(\operatorname{chps} \mathrm{c} \mathrm{n} 1) /$ select $<$

[ aselect

sel syn (von-dat-pp-syn davon-dass-syn davon-wh-syn)] $\rangle$

example $\langle$ "Die Manager sind nicht unabhängig von den Arbeitern." $\rangle]]$

$\%$ 'The managers are not independent from

$\%$ the workers.'

Figure 1: Simplified lexicon entries for the verb prahlen, the noun Gegenstück, and the adjective unabhängig. 
aus.origm examples: eine Platte aus Kupfer ('a plate out of copper'), ...

$\operatorname{sort}(\mathrm{c} 1)=\operatorname{co} \wedge \operatorname{sort}(\mathrm{c} 2)=\mathrm{s} \rightarrow($ origm $\mathrm{c} 1 \mathrm{c} 2)$

auf.attr_language examples: ein Artikel auf Englisch ('an article in English'), ...

$((\operatorname{sort}(\mathrm{c} 1)=\mathrm{o} \wedge \operatorname{info}(\mathrm{c} 1)=+) \vee \operatorname{sort}(\mathrm{c} 1)=\mathrm{ad}) \wedge(\operatorname{sub} \mathrm{c} 2$ "sprache.1.1")

$\rightarrow(\operatorname{attr} \mathrm{c} 1 \mathrm{c} 3) \wedge($ val c3 c2) $\wedge($ sub c3 "sprache.1.1")

Figure 2: Examples of PP interpretation rules; $\mathrm{c} 1$ refers to the PP's mother constituent, $\mathrm{c} 2$ to the preposition's complement; features are explained in Sect. 3.

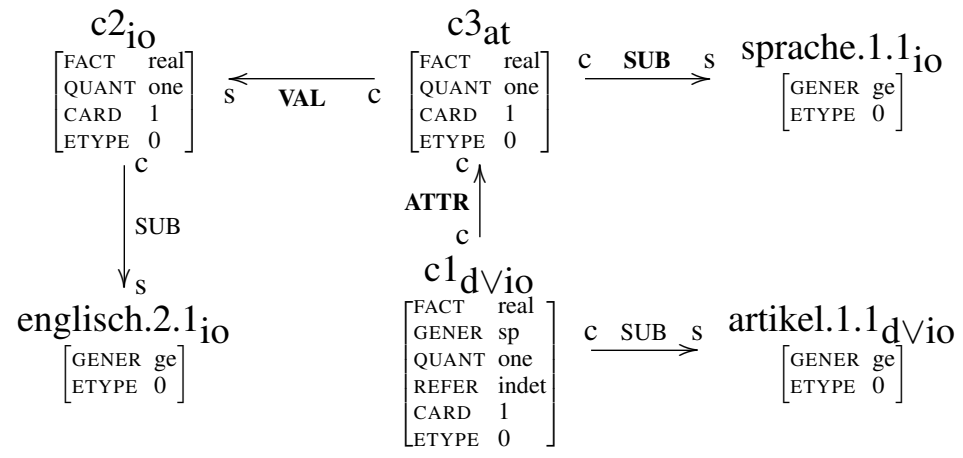

Figure 3: Semantic network for the noun phrase ein Artikel auf Englisch ('an article in English'); the relations in bold face and $c 3$ stem from the conclusion of the PP rule auf.attr_language shown in Fig. 2.

tation. ${ }^{7}$ This knowledge acts as the training set for a machine learning component that disambiguates attachment and interpretation of PPs (see Sect. 5).

\section{Preposition Interpretation within Semantic Parsing}

All the knowledge resources described in Sect. 4 are used by the parser to determine the correct interpretation of prepositions. Furthermore, PP attachment ambiguities are resolved on the basis of possible interpretations. The complement information (valency frames) in the lexicon licenses possible complement interpretations, the PP interpretation rules (combined with the adjunct information in the lexicon) license possible adjunct interpretations. In case of alternatives, they are disambiguated using statistics derived from the annotated PP corpus and a whole range of preference scores. ${ }^{8}$

The statistical data is represented in the form of a multidimensional back-off model. Each alternative is described by the rule name, the semantics of

\footnotetext{
${ }^{7}$ In case of so-called systematic ambiguities, both attachments have been classified as valid. Moreover, two readings were considered as equally likely in some cases.

${ }^{8}$ Classical rule-based approaches often apply some sort of decision algorithm to disambiguate such cases; see e.g. (Hirst, 1987).
}

the preposition's complement, and the semantics of the possible syntactic head. If no exact match is found in the disambiguation statistics the number of considered alternatives and the granularity of the description of an alternative are reduced by backing off in these two orthogonal dimensions; see (Hartrumpf, 2003; Hartrumpf, 1999) for details.

\section{Evaluation}

\subsection{Intrinsic Evaluation}

Experiments showed that $24.2 \%$ of verb complement interpretations are equally well produced by adjunct rules (see column 5 in Table 3). Nevertheless the PP interpretation disambiguation task profits from complement information because in many of these overlap cases more than one PP interpretation was possible. Also the PP attachment disambiguation task can benefit from the complement vs. adjunct distinction because complementhood is a strong indicator of the correct attachment place. A third argument for having such complement information is that it is important to model all roles belonging to a concept on the cognitive level; this can be easily realized by a one-to-one correspondence between cognitive roles and complements in the lexicon. Table 3 shows the number 
Table 3: PP complements in the lexicon. Mixed PP complement means that the role can be syntactically realized as a PP or an NP. Reg. compl. means that the complement semantics specified in the lexicon is equally well produced by some PP interpretation rule and therefore viewed as being regular.

\begin{tabular}{lrrrrrr}
\hline cat. & lexemes & PP compl. & $\begin{array}{c}\text { lexemes with PP } \\
\text { compl. }\end{array}$ & reg. compl. & mixed PP compl. & $\begin{array}{c}\text { lexemes with } \\
\text { mixed PP compl. }\end{array}$ \\
\hline $\mathrm{V}$ & 7006 & 1690 & 1616 & $24.2 \%$ & 105 & 100 \\
$\mathrm{n}$ & 13111 & 720 & 684 & $5.6 \%$ & 3750 & 2393 \\
\hline
\end{tabular}

of lexemes of a given category with some PP complements and the total number of PP complements for verbs and nouns in our lexicon. The percentage of regular complements $(24.2 \%)$ is significantly higher than the corresponding token value in the QA@CLEF corpus (17.5\%, see Table 2). This indicates that regular complements often have a more optional character than irregular complements. Also in this respect, regular complements resemble adjuncts.

The preposition interpretation method achieves between $84 \%$ and $89 \%$ correctness for the six prepositions supported by the hand-tagged PP corpus; for prepositions without annotated corpus data, the performance seems to drop by around 10 to 20 percent points.

\subsection{Extrinsic Evaluation}

One important application using the parser and the preposition interpretation described above is InSicht, a QA system for German text collections (Hartrumpf, 2005). To measure the impact of a deep preposition interpretation, the QA system was run twice: with and without the PP interpretation presented above. For the latter, each interpretation of a PP with preposition $p$, an NP $c 2$, and syntactic head $c 1$ was replaced by an edge with the unique artificial relation PP.p, e.g. the aus-PP rule in Fig. 2 would contain the conclusion (PP.AUS $c 1 c 2$ ). The QA system was evaluated on the German questions from the question answering track at CLEF (QA@ CLEF) of the years 2004 and 2005. Surprisingly, the PP interpretation with unique artificial relations caused no significant performance drop. A closer look at all questions from QA@CLEF 2004 involving PPs revealed that the documents with answers almost always contained the same prepositions as the corresponding questions. Therefore we tried more difficult (and often more realistic) questions with different prepositions (and verbs or nouns). Only natural and (nearly) equivalent paraphrases were allowed. For the PP questions where the QA system delivered correct answers, 14 paraphrases were written to test the positive impact of transforming surface prepositions to their correct meaning.

The evaluation of PP semantics was then performed using the paraphrases instead of the original questions. For $86 \%$ of all paraphrases, the correct answer was still found when the more distant paraphrase was used as the question for the QA system; with the artificial relations for PPs, only $14 \%$ of the paraphrases were answered correctly. This indicates clearly that NLP applications like semantic QA systems benefit from a good preposition interpretation. The paraphrases that could not be answered by the QA system with PP interpretation would need more advanced reasoning techniques to work correctly.

Some paraphrases involved PP adjuncts. For example, the QA@CLEF question qa04_055 is given as example (5):

(5) In welchem Jahr wurde Nelson Mandela In which year was Nelson Mandela geboren?

born?

'In which year was Nelson Mandela born?'

As the documents contain the correct answer in the form of a PP with the same preposition as in the question (in), shallow approaches (and also our QA system with the artificial relations) can answer this question correctly. But the paraphrase (6) requires that question and documents, which differ on the surface (in-PP vs. interrogative Wann), are transformed to the same representation (expressing a temporal relation).

(6) Wann wurde Nelson Mandela geboren?

When was Nelson Mandela born? 'When was Nelson Mandela born?'

There were also some cases illustrating the im- 
portance of a homogenous transition between the semantics of PP adjuncts and the semantics of PP complements. Example (7) (qa04_027) contains an interrogative involving für, which is specified as a complement of the verb anklagen ('accuse') in the current version of HaGenLex.

\author{
Wofür wurde Aldrich H. Ames \\ What-for was Aldrich H. Ames \\ angeklagt? \\ accused? \\ 'For what was Aldrich H. Ames accused?'
}

But in paraphrases like (8), the same entity appears as an adjunct of the verb.
Weswegen
wurde Aldrich $\mathrm{H}$.
What-GEN-because-of was Aldrich $\mathrm{H}$.
Ames angeklagt?
Ames accused?
'Because of what was Aldrich H. Ames ac- cused?'

The correct answer is still found for the paraphrase because both questions and the relevant document sentences contain the same semantic representation (here, a single relation of justification).

All these paraphrases are examples of increased recall. But also the precision of the QA system is improved because preposition sense mismatches between question and documents can be detected.

\section{Conclusion and Future Work}

We have presented a unified approach to the problem of automatic preposition interpretation. The extrinsic evaluation result in the context of a QA system encourages us to continue work in the following directions. The PP interpretation rules are to be transferred to other languages. We have already started for English. The transfer on the level of preposition readings (or PP rules) is much easier than translating prepositions. Moreover, the coverage and quality of all three knowledge resources should be further extended. For example, we want to analyze why certain PP interpretation rules have rarely succeeded during corpus parsing. Extrinsic evaluations on larger and more difficult question sets for QA systems and evaluations in other NLP applications might help to focus further research.

\section{References}

Timothy Baldwin. 2005. Looking for prepositional verbs in corpus data. In Proc. of the 2 nd ACL-SIGSEM Workshop on Prepositions, Colchester, UK.

Sven Hartrumpf, Hermann Helbig, and Rainer Osswald. 2003. The semantically based computer lexicon HaGenLex - Structure and technological environment. Traitement automatique des langues, 44(2):81-105.

Sven Hartrumpf. 1999. Hybrid disambiguation of prepositional phrase attachment and interpretation. In Proc. of EMNLP/VLC-99, pages 111-120, College Park, Maryland.

Sven Hartrumpf. 2003. Hybrid Disambiguation in Natural Language Analysis. Der Andere Verlag, Osnabrück, Germany.

Sven Hartrumpf. 2005. University of Hagen at QA@CLEF 2005: Extending knowledge and deepening linguistic processing for question answering. In Carol Peters, editor, Results of CLEF 2005. Centromedia, Wien.

Hermann Helbig. 2006. Knowledge Representation and the Semantics of Natural Language. Springer.

Graeme Hirst. 1987. Semantic Interpretation and the Resolution of Ambiguity. Cambridge University Press.

Todd A. Letsche and Michael W. Berry. 1997. Largescale information retrieval with latent semantic indexing. Information Sciences, 100(1-4):105-137.

Johannes Leveling and Sven Hartrumpf. 2005. University of Hagen at CLEF 2004: Indexing and translating concepts for the GIRT task. In C. Peters et al., editors, Multilingual Information Access for Text, Speech and Images, LNCS 3491, pages 271-282. Springer.

Johannes Leveling and Hermann Helbig. 2002. A robust natural language interface for access to bibliographic databases. In Nagib Callaos et al., editors, Proc. of SCI 2002, volume XI, pages 133-138, Orlando, Florida.

Johannes Leveling. 2005. University of Hagen at CLEF 2005: Towards a better baseline for NLP methods in domain-specific information retrieval. In Carol Peters, editor, Results of CLEF 2005. Centromedia, Wien.

Kenneth C. Litkowski and Orin Hargraves. 2005. The preposition project. In Proc. of the 2nd ACLSIGSEM Workshop on Prepositions, Colchester, UK.

Kenneth C. Litkowski. 2002. Digraph analysis of dictionary preposition definitions. In Proc. of the ACL Workshop on Word Sense Disambiguation, pages 916, Philadelphia, Pennsylvania. 
Tom O'Hara and Janyce Wiebe. 2003. Preposition semantic classification via Penn Treebank and FramNet. In Proc. of CoNLL-2003. Edmonton, Canada.

Rainer Osswald, Hermann Helbig, and Sven Hartrumpf. 2006. The representation of German prepositional verbs in a semantically based computer lexicon. In Proc. of LREC 2006, Genoa, Italy. To appear.

Gisa Rauh. 1993. On the grammar of lexical and non-lexical prepositions in English. In Cornelia Zelinski-Wibbelt, editor, The Semantics of Prepositions, pages 99-150. Mouton de Gruyter.

Patrick Saint-Dizier. 2005. PrepNet: A framework for describing prepositions: Preliminary investigation results. In Proc. of IWCS 6, Tilburg.

Cornelia Zelinski-Wibbelt, editor. 1993. The Semantics of Prepositions. Mouton de Gruyter.

\section{Appendix: Elements of the MultiNet Formalism}

Table 4: Part of the hierarchy of ontological sorts

\begin{tabular}{|c|c|c|}
\hline \multicolumn{3}{|c|}{ entity [ent] } \\
\hline \multicolumn{3}{|c|}{ object $[o]$} \\
\hline \multicolumn{3}{|c|}{ concrete object $[\mathrm{co}]$} \\
\hline & discrete object $[d]$ & house, apple, tiger \\
\hline & substance $[s]$ & milk, honey, iron \\
\hline \multicolumn{3}{|c|}{ abstract object $[a b]$} \\
\hline & attribute $[a t]$ & height, weight \\
\hline & relationship $[\mathrm{re}]$ & causality \\
\hline & ideal object $[$ io $]$ & religion, justice \\
\hline & modality $[\mathrm{mo}]$ & necessity, intention \\
\hline & situational object $[a b s]$ & race, robbery \\
\hline \multicolumn{3}{|c|}{$\cdots$} \\
\hline \multicolumn{3}{|c|}{ situation $[\mathrm{si}]$} \\
\hline \multicolumn{3}{|c|}{ dynamic situation $[d y]$} \\
\hline & action $[d a]$ & write, sing, sell \\
\hline & happening $[d n]$ & rain, decay \\
\hline & static situation $[s t]$ & stand, be ill \\
\hline \multicolumn{3}{|c|}{ situational descriptor $[s d]$} \\
\hline \multicolumn{3}{|r|}{ yesterday, Monday } \\
\hline \multicolumn{3}{|c|}{ location $[l]$} \\
\hline \multicolumn{3}{|c|}{$\ldots$} \\
\hline \multicolumn{3}{|c|}{ quality $[q l]$} \\
\hline \multicolumn{3}{|c|}{ property $[p]$} \\
\hline & total quality $[t q]$ & dead, empty, green \\
\hline & gradable quality $[g q]$ & friendly, expensive \\
\hline \multicolumn{3}{|c|}{$\cdots$} \\
\hline \multicolumn{3}{|r|}{$\begin{array}{l}\text { inverse, equivalent, } \\
\text { similar }\end{array}$} \\
\hline \multicolumn{3}{|c|}{ functional quality $[f q]$} \\
\hline & operational quality $[o q]$ & fourth, last, next \\
\hline & associative quality $[a q]$ & chemical \\
\hline \multicolumn{3}{|c|}{ quantity $[q n]$} \\
\hline & quantificator $[q f]$ & one, many, several \\
\hline & measurement $[\mathrm{m}]$ & two litres \\
\hline & duator $[g r]$ & very, quite \\
\hline & $\cdots$ & \\
\hline
\end{tabular}

Table 5: Strongly abbreviated description of relations used in this paper.

\begin{tabular}{|c|c|c|}
\hline Rel. & Signature & Short Characteristics \\
\hline AGT & {$[a b s \cup s i] \times o$} & C-Role-Agent \\
\hline ATTCH & $\begin{array}{l}{[o \backslash a t] \times} \\
{[o \backslash a t]}\end{array}$ & Attachments of objects \\
\hline ATTR & {$[l \cup o \cup t] \times a t$} & $\begin{array}{l}\text { Specification of an at- } \\
\text { tribute }\end{array}$ \\
\hline AVRT & {$[a d \cup d y] \times o$} & $\begin{array}{l}\text { C-Role - Averting/Turn- } \\
\text { ing away from an object }\end{array}$ \\
\hline CAUS & $\begin{array}{l}{\left[a b s^{\prime} \cup s i^{\prime}\right] \times} \\
{\left[a b s^{\prime} \cup s i^{\prime}\right]}\end{array}$ & $\begin{array}{l}\text { Relation between cause } \\
\text { and effect (causality) }\end{array}$ \\
\hline CHPS & {$[p \cup r q] \times[a s \cup s t]$} & $\begin{array}{l}\text { Change of sorts: Property } \\
\text { - State }\end{array}$ \\
\hline DIRCL & {$[o \cup s i] \times l$} & $\begin{array}{l}\text { Relation specifying a di- } \\
\text { rection }\end{array}$ \\
\hline DUR & $\begin{array}{l}{[o \cup s i] \times} \\
{[a b s \cup s i \cup t \cup t a]}\end{array}$ & $\begin{array}{l}\text { Relation specifying a } \\
\text { temporal extension }\end{array}$ \\
\hline ELMT & $e n t \times e n t$ & Element relation \\
\hline EQU & $e n t \times e n t$ & $\begin{array}{l}\text { Equality/Equivalence re- } \\
\text { lation }\end{array}$ \\
\hline FIN & $\begin{array}{l}{[o \cup s i \cup t] \times} \\
{[a b s \cup s i \cup t \cup t a]}\end{array}$ & $\begin{array}{l}\text { Relation specifying the } \\
\text { temporal end }\end{array}$ \\
\hline INSTR & {$[a b s \cup s i] \times c o$} & C-Role - Instrument \\
\hline LOC & {$[o \cup s i] \times l$} & $\begin{array}{l}\text { Relation specifying the } \\
\text { location }\end{array}$ \\
\hline MCONT & {$[o \cup s i] \times[o \cup s i]$} & $\begin{array}{l}\text { C-Role - Relation be- } \\
\text { tween a mental process } \\
\text { and its content }\end{array}$ \\
\hline OBJ & $\begin{array}{l}{[a b s \cup s i] \times} \\
{[o \cup s i]}\end{array}$ & $\begin{array}{l}\text { C-Role - Neutral object } \\
\text { of a situation }\end{array}$ \\
\hline ORIGM & $\mathrm{co} \times \mathrm{co}$ & $\begin{array}{l}\text { Relation specifying the } \\
\text { material origin }\end{array}$ \\
\hline ORNT & {$[a b s \cup s i] \times o$} & $\begin{array}{l}\text { C-Role - Orientation of } \\
\text { a situation toward some- } \\
\text { thing }\end{array}$ \\
\hline PARS & $\begin{array}{l}{[c o \cup i o \cup l \cup t a] \times} \\
{[c o \cup i o \cup l \cup t a]}\end{array}$ & Part-whole relationship \\
\hline PROP & $o \times p$ & $\begin{array}{l}\text { Relation between object } \\
\text { and property }\end{array}$ \\
\hline SCAR & {$[a s \cup s t] \times o$} & $\begin{array}{l}\text { C-Role - Carrier of a } \\
\text { state }\end{array}$ \\
\hline SSPE & $\begin{array}{l}{[a s \cup s t] \times} \\
{[m \cup o \cup s i]}\end{array}$ & $\begin{array}{l}\text { C-Role - Entity specify- } \\
\text { ing a state }\end{array}$ \\
\hline STRT & $\begin{array}{l}{[o \cup s i \cup t] \times} \\
{[a b s \cup s i \cup t \cup t a]}\end{array}$ & $\begin{array}{l}\text { Relation specifying the } \\
\text { temporal begin }\end{array}$ \\
\hline SUB & $o \times \bar{o}$ & $\begin{array}{l}\text { Relation of concep- } \\
\text { tual subordination (for } \\
\text { objects) }\end{array}$ \\
\hline SUBM & $e n t \times e n t$ & $\begin{array}{l}\text { Relation of set subsump- } \\
\text { tion }\end{array}$ \\
\hline SUBS & $\begin{array}{l}{[a b s \cup s i] \times} \\
{[\overline{a b s} \cup \overline{s i}]}\end{array}$ & $\begin{array}{l}\text { Relation of conceptual } \\
\text { subordination (for situa- } \\
\text { tions) }\end{array}$ \\
\hline TEMP & $\begin{array}{l}{[o \cup s i \cup t] \times} \\
{[a b s \cup s i \cup t \cup t a]}\end{array}$ & $\begin{array}{l}\text { Relation specifying the } \\
\text { temporal embedding of a } \\
\text { situation }\end{array}$ \\
\hline VAL & $\begin{array}{l}a t \times \\
{[o \cup p \cup q n \cup t]}\end{array}$ & $\begin{array}{l}\text { Relation between an at- } \\
\text { tribute and its value }\end{array}$ \\
\hline
\end{tabular}

